\title{
A special issue on the Boris V. Gnedenko Centennial
}

\author{
Larisa G. Afanasyeva - Dmitry Korshunov • \\ Dmitry Shabanov
}

This special issue contains a selection of papers written by the participants of the international conference Probability Theory and Its Applications which was held on 26-30 June 2012 at Lomonosov Moscow State University, Russia. It was organised by Moscow State University jointly with Steklov Mathematical Institute and Moscow Institute of Electronics and Mathematics.

The conference was dedicated to the Commemoration of the Centennial of the outstanding probabilist and specialist in queueing theory Boris Vladimirovich Gnedenko (01.01.1912-27.12.1995). More than 200 talks were given; they presented recent developments in the fields of primary interest to Gnedenko: limit theorems, stochastic extreme value theory, queueing theory, mathematical reliability theory and actuarial mathematics. The conference was attended by many experts in these areas, in particular, by many colleagues and former students of B. V. Gnedenko.

The special issue includes an invited overview paper by Igor N. Kovalenko and five research papers based on presentations given in the section Queueing Theory of the conference.

The Invited Paper Boris Vladimirovich Gnedenko: A Classical Scholar in Probability, Statistics, Queueing and Reliability is written by Igor N. Kovalenko, a well-known expert in queueing theory. He was one of the closest friends of B. V. Gnedenko and

\footnotetext{
L. G. Afanasyeva · D. Shabanov

Lomonosov Moscow State University, Moscow, Russia

e-mail: 1.g.afanaseva@yandex.ru

D. Shabanov

e-mail: dm.shabanov.msu@gmail.com

D. Korshunov $(\varangle)$

Sobolev Institute of Mathematics, Novosibirsk 630090, Russia

e-mail: Korshunov@math.nsc.ru
} 
one of his most frequent co-authors. The overview paper provides knowledge on Gnedenko's personality, his life in mathematics and mathematics in his life.

The next paper Coupling method for asymptotic analysis of queues with regenerative input and unreliable server written by Larisa G. Afanasyeva and Elena Bashtova starts with a discussion on regenerative flows and description of their properties; this kind of flow is a natural generalisation of many other flows (semi Markov, Markov modulated, Markov arrival, etc.). Following the coupling techniques, the authors investigate ergodicity properties of a single server queueing system with regenerative input flow and unreliable server. They also present some approximation results in the case of heavy traffic.

In his paper Clock synchronization in symmetric stochastic networks, Anatoly Manita considers a stochastic model of clock synchronisation in a wireless network of $N$ sensors interacting with a single accurate time server. The sensor clocks are not perfect and are assumed to follow Brownian motions. The main results are concerned with the asymptotic behaviour of the synchronisation error on different time scales $t=t_{N} \rightarrow \infty, N \rightarrow \infty$, in terms of the average value of the squares of offsets between clocks. The author also discusses the existence of phase transitions and establishes exact time scales for which an effective clock synchronisation of the system takes place.

The paper On the rate of convergence for infinite server Erlang-Sevastyanov's problem by Alexander Veretennikov is related to Erlang's problem of description of the stationary distribution in the classical telephone system with non-exponential service times. A good tool for proving the total variation convergence to the stationary distribution is the celebrated B. A. Sevastyanov's ergodic theorem which can be extended even for the case of infinite number of servers. The author proves a polynomial rate of convergence to the stationary distribution in the system with infinite number of servers under certain moment conditions on the service time distribution.

The Perturbation bounds and truncations for a class of Markovian queues are studied in the paper by Alexander Zeifman, Victor Korolev, Yacov Satin, Anna Korotysheva and Vladimir Bening. They consider time-inhomogeneous Markovian queueing models with batch arrivals and group services; in the context of birth-death models, such kinds of processes go back to B. V. Gnedenko and his colleagues. For given two time-inhomogeneous Markovian queueing models, the authors derive asymptotic in time upper bounds for the distance between (i) their distributions and (ii) mathematical expectations of their queue-length processes in terms of the distance between input characteristics of the queueing models.

The paper On ergodicity conditions in a polling model with Markov-modulated input and state-dependent routing by Andrei V. Zorine deals with a Markovian polling system with switchover times and state-dependent server routing. The Poisson input flows are modulated by a random external environment with two states. The author considers a continuous-time Markov chain which describes the dynamics of the server, the sizes of the queues and the states of the environment. He obtains a sufficient condition for ergodicity of this continuous time Markov chain. 